\title{
Striving to ensure new oncological therapies do not put added strain on healthcare resources
}

\author{
Neal D. Shore, MD, FACS \\ Medical Director, CPI, Carolina Urologic Research Center, Myrtle Beach, SC, United States
}

Cite as: Can Urol Assoc J 2018;12(12):377. hitrp://dx.doi.org/10.5489/cuai.5756

See related article on page 370

S ymptomatic skeletal events are indeed a burden to both aggrieved prostate cancer patients, as well as to the resources of the healthcare system. Saad et al have performed a retrospective data analysis of $\mathrm{mCRPC}$ patients form 2006-20013, reviewing the use of bone-targeted therapies (BTTs), mostly zoledronic acid (ZA), at three well-known and highly respected uro-oncology centres located in Montreal (Centre Hospitalier de I'Université de Montréal [CHUM]), Toronto (Princess Margaret Cancer Centre [PMCC]), and Vancouver (Vancouver General Hospital [VGH]).

Despite the universal acceptance that BTTs reduce skeletal-related events (SREs) - from well-designed, prospective phase 3 trials demonstrating the delay of SRE when using BTT, whether ZA or denosumab - the review from these three leading Canadian centres suggests that only $51 \%$ of appropriate metastatic castrate-resistant prostate cancer ( $\mathrm{mCRPC}$ ) patients received an approved BTT, and notably at $\mathrm{VGH}$, only $24 \%$ patients did.

mCRPC patients receiving BTT had prolonged times to symptomatic SREs, which was also associated with decreased healthcare resource utilization (HRU). In fact, there was anywhere from a two- to three-and-a-half-fold increase in HRU when a suboptimal BTT use was initiated. Specifically, the authors note and suggest that an every 3-4-week schedule of ZA administration resulted in better outcomes in comparison to a schedule of every 3-4 months; presumably, the latter was chosen for cost benefit and patient convenience, as well as some prior smaller trials suggesting the reduction in therapy schedule was clinically comparable to the approved schedule per the drug-labelled indication. Of note, the schedule for BTT administration for VGH is not provided. Assuredly, this paper confirms the well-documented literature that symptomatic (and ultimately asymptomatic) SREs sustain a significant health economic burden upon the healthcare system, and the authors cite that there still exists a paucity of clinical data with correlative economic data

that substantiates this seemingly intuitive notion, and thus the value of their paper.

The analysis has some limitations, which the authors cite: potential chart (presumably both paper and electronic) data deficits; a review of only mCRPC patients who died of prostate cancer-specific mortality and who also underwent hospitalization, which may have overestimated total attributable costs; uncertainty of variations and spectrum disease burden for the population reviewed; the patient population comorbidities; and importantly, the use of other approved mCRPC anti-neoplastic therapies, which may have ameliorated SRE findings. In retrospective chart pull analyses, there is inherent difficulty of accuracy and of ascertaining abstraction bias of data among and within centres, and thus, a potential risk of appropriately assigning actual HRUs. Nonetheless, based on numerous other trials and analyses, as well as guideline recommendations, BTT should be discussed and used for the vast majority of $\mathrm{mCRPC}$ patients when indicated. Further to BTT, other important SRE preventative measures continue to be underused, as does avoidance of a sedentary lifestyle, nutritional management, vitamin and calcium supplementation, family history assessment for bone fragility, etc.

The authors conclude that the development of symptomatic skeletal events and the use of BTT significantly varied among three major uro-oncology centres within Canada and were associated with negative HRU implications. In effect, their analysis should inspire all centres to review their symptomatic skeletal event prevention strategies, especially considering all available BTT and the correlative impact within and upon the attribution of the health economic environment for their institution, as we further strive to ensure that new and improved oncological therapies and their additional costs can sufficiently demonstrate HRU savings.

Competing interests: Dr. Shore has been an advisory board member for Amgen, Astellas, AstraZeneca, Bayer, BMS, Dendreon, Ferring, Janssen, Merck, Pizer, Sanofi, and Tolmar; and has participated in clinical trials supported by Amgen, Astellas, AstraZeneca, Bayer, BMS, Dendreon, Ferring, Janssen, Merck, Pizer, Sanofi, and Tolmar.

Correspondence: Dr. Neal Shore, Medical Director, CPI, Carolina Urologic Research Center, Myrtle Beach, SC, United States; NShore@gsuro.com 